\title{
Efeito da idade de exposição de novilhas à reprodução sobre estimativas de herdabilidade da idade ao primeiro parto em bovinos Nelore
}

[Effect of age of exposure of heifers to reproduction on heritability estimates for age at first calving in Nelore cattle]

\author{
L.T. Dias $^{1}$, L. EL Faro ${ }^{2}$, L.G. Albuquerque ${ }^{3}$ \\ ${ }^{1}$ Doutoranda do Departamento de Zootecnia - FCAV-UNESP \\ Via de Acesso Prof. Paulo Donato Castellane \\ 14884-900 - Jaboticabal, SP \\ ${ }^{2}$ Instituto de Zootecnia de Ribeirão Preto - SP - APTA \\ ${ }^{3}$ Departamento de Zootecnia UNESP - Jaboticabal
}

\begin{abstract}
RESUMO
Estimou-se a herdabilidade da idade ao primeiro parto (IPP) a partir de quatro conjuntos com diferentes tipos de informação. A primeira estimativa (IPP1) foi realizada com as novilhas $(n=6.222)$ expostas apenas na estação "normal", ou seja, aos 24 meses de idade. No segundo conjunto (IPP2), fizeram parte do arquivo todas as fêmeas $(n=15.746)$ com informação da IPP. No terceiro (IPP3), estimou-se a herdabilidade de um grupo mais restrito de novilhas $(n=9.524)$, as quais tiveram manejo diferenciado na fazenda; todas as fêmeas pertencentes a este grupo foram expostas mais cedo à reprodução, aproximadamente 18 meses de idade (estação de monta "antecipada"). Para o quarto grupo (IPP4), usouse o arquivo completo de dados, ou seja, os registros de todas as fêmeas nascidas no rebanho $(\mathrm{n}=40.954)$. Foram considerados no modelo o efeito aleatório de animal e os efeitos fixos de grupo contemporâneo e os efeitos linear e quadrático de idade da mãe ao parto. As herdabilidades estimadas para IPP1, IPP2, IPP3 e IPP4 foram, respectivamente, 0,$00 ; 0,11 ; 0,20$ e 0,36 .
\end{abstract}

Palavras-chave: gado de corte, herdabilidade, precocidade sexual

\begin{abstract}
Heritability estimates of age at first calving (AFC) were obtained from four different data sets from Nellore heifers. The first set (AFCI) had information about heifers $(n=6,222)$ which were exposed only at 24 months of age. The second (AFC2) data set used all heifers $(n=15,746)$ with information about AFC. The third set (AFC3) included a restricted number of heifers $(n=9,524)$, which were exposed earlier to reproduction at 18 months of age. The fourth set (AFC4) had information about all heifers born on herd $(n=40,954)$. Analyses included as fixed effects the contemporary group and linear and quadratic effects of age of dam. Estimates of heritability for AFC1, AFC2, AFC3 and AFC4 were, respectively, 0.00, 0.11, 0.20 and 0.36. Age at first calving was affected by reproductive management.
\end{abstract}

Keywords: beef cattle, heritability, sexual precocity

\section{INTRODUÇÃO}

Recentemente vários trabalhos foram desenvolvidos no Brasil com o objetivo de aumentar a eficiência produtiva da pecuária de corte (Teixeira, 1997; Gressler, 1998, Pereira, 2001 e 2002; Eler, 2002). Melhorar a precocidade sexual das fêmeas pode ser uma das alternativas para se alcançar maior sucesso na atividade.

Recebido para publicação em 21 de fevereiro de 2003

Recebido para publicação, após modificações, em 30 de janeiro de 2004

E-mail:laila@fcav.unesp.br 
$\mathrm{Na}$ literatura discute-se as vantagens e desvantagens em iniciar a vida reprodutiva das novilhas mais cedo. Segundo Short et al. (1994), entre as principais vantagens em emprenhar as novilhas mais jovens estão: menor tempo para se obter retorno do investimento, aumento da vida reprodutiva da vaca e aumento do número de bezerros.

A característica idade ao primeiro parto pode ser facilmente medida, não exige custos extras para sua obtenção e vem sendo utilizada como critério de seleção para precocidade sexual. Alguns autores relataram que as características reprodutivas têm, geralmente, baixa herdabilidade (Bourdon e Brinks, 1982; Toelle e Robison, 1985). No Brasil, Martins Filho e Lôbo (1991), Mercadante (1995) e Gressler (1998) estimaram herdabilidades para a idade ao primeiro parto de 0,$19 ; 0,25$ e 0,00 , respectivamente, para animais da raça Nelore.

A idade ao primeiro parto é uma característica sujeita à influência do manejo reprodutivo adotado na fazenda. A maioria dos produtores pré determina a idade ou o peso como condição para o início da vida reprodutiva. Dessa forma, a identificação das novilhas precoces fica comprometida e as estimativas de herdabilidade para essa característica são baixas devido à baixa variabilidade existente. Com a mudança em algumas medidas de manejo é possível verificar sua influência na identificação dos animais precoces e nas estimativas de herdabilidade para essa característica.

O objetivo do presente trabalho foi observar o impacto dos diferentes tipos de informação sobre as estimativas de herdabilidade para a idade ao primeiro parto em novilhas da raça Nelore.

\section{MATERIAL E MÉTODOS}

Foram analisados registros de idade ao primerio parto de animais da raça Nelore nascidos entre 1984 e 1996, pertencentes à Agropecuária Jacarezinho Ltda., situada no município de Valparaíso - SP. Até o ano de 1989, o manejo reprodutivo das novilhas era feito com uma estação de monta denominada "normal", com duração aproximada de 70 dias, entre os meses de novembro e janeiro. A partir de 1990, foi iniciado um trabalho de antecipação da estação de monta para as novilhas de 18-20 meses, posteriormente para os animais com 17-19 meses de idade e, atualmente, para novilhas entre 16-18 meses de idade. A estação de monta "antecipada" é realizada entre os meses de abril e maio e apresenta duração de, aproximadamente, 60 dias. Os animais que não concebem na estação de monta "antecipada" têm uma segunda oportunidade na estação "normal", e os que não concebem nesta segunda estação são descartados do rebanho.

Foram estimadas herdabilidades para idade ao primeiro parto (IPP) utilizando-se quatro conjuntos de dados (IPP1, IPP2, IPP3 e IPP4) que diferiram quanto ao tipo de informação para a característica estudada. Para IPP1 consideraram-se as novilhas expostas pela primeira vez apenas aos 24 meses de idade, aproximadamente, ou seja, todos os animais que pariram antes de 1990. Para IPP2 foram incluídas todas as fêmeas que tinham informação do primeiro parto, independentemente de terem tido oportunidade de cobrição em uma ou duas estações de monta. No conjunto IPP3, permaneceram no arquivo apenas as fêmeas expostas mais cedo à reprodução. Assim, o arquivo IPP3 continha apenas as fêmeas que participaram da estação de monta antecipada, ou seja, pariram a partir do início da década de 90. Para IPP4 consideraram-se todas as novilhas nascidas no rebanho, mesmo as que não pariram. Para as que não tinham informação da idade ao primeiro parto, considerou-se a idade de sete anos apenas para que elas fizessem parte das análises. Os arquivos de dados gerados para IPP1, IPP2, IPP3 e IPP4 contaram com 6.222, $15.746,9.524$ e 40.954 informações, respectivamente.

Para realização das análises foi utilizado um modelo misto, com efeito aleatório de animal e os efeitos fixos grupo contemporâneo, como classe, e idade da vaca, mãe do animal analisado, como covariável. O grupo contemporâneo (GC) foi definido pelas seguintes variáveis: fazenda, ano, estação e grupo de manejo ao nascimento, grupo de manejo à desmama e ao sobreano, e tipo de cobertura (monta natural por reprodutores múltiplos, monta controlada ou inseminação artificial).

O modelo misto geral utilizado pode ser representado na forma matricial como: 
$y=X \beta+Z a+e$, em que:

$\mathrm{y}=$ vetor de variáveis dependentes;

$\beta=$ vetor de efeitos fixos;

$a=$ vetor de valores genéticos aditivos dos animais;

$e=$ vetor de efeitos residuais;

$X e Z$ são matrizes de incidência respectivas para cada efeito.

As pressuposições em relação aos componentes do modelo são:

$\mathrm{E}(y)=X \beta, E(a)=0$ e $E(e)=0$

$\operatorname{Var}\left[\begin{array}{l}\mathrm{a} \\ \mathrm{e}\end{array}\right]=\left[\begin{array}{ll}\mathrm{G} & 0 \\ 0 & \mathrm{R}\end{array}\right]$, em que:

$\mathrm{G}=$ matriz de variância genética aditiva, obtida por A $\sigma_{a}^{2}$;

$\mathrm{A}=$ matriz de parentesco;

$R=$ matriz de variância residual, obtida por $\mathrm{I} \sigma_{e}^{2}$;

$\mathrm{I}=$ matriz identidade;

$\sigma_{a}^{2}=$ variância genética aditiva direta;

$\sigma_{e}^{2}=$ variância residual.

Para estimação dos componentes de variância utilizou-se o método da máxima verossimilhança restrita, empregando-se $\mathrm{o}$ processo não derivativo. Foram aplicados modelos animais utilizando o algoritmo desenvolvido por Boldman et al. (1993), disponível no pacote MTDFREML.

\section{RESULTADOS E DISCUSSÃO}

As estimativas de herdabilidade para IPP variaram de 0,00 a 0,36 de acordo com o tipo de informação utilizada (Tab. 1).

Tabela 1. Estimativas de omponentes de variância e coeficientes de herdabilidade para idade ao primeiro parto (IPP) de animais Nelore, em diferentes conjuntos de dados

\begin{tabular}{lcccc}
\hline & IPP1 & IPP2 & IPP3 & IPP4 \\
\hline$\sigma_{\mathrm{a}}{ }^{2}$ & 0,026 & 594,11 & $1.365,28$ & $109.104,88$ \\
$\sigma_{\mathrm{e}}{ }^{2}$ & 537,80 & $4.610,53$ & $5.389,19$ & $197.413,08$ \\
$\mathrm{~h}^{2}$ & 0,00 & 0,11 & 0,20 & 0,36 \\
\hline$\sigma_{\mathrm{a}}{ }^{2}=$ variância do & efeito & genético aditivo & direto, $\sigma_{\mathrm{e}}{ }^{2}=$ \\
variância residual, $\mathrm{h}^{2}=$ coeficiente de herdabilidade.
\end{tabular}

Para IPP1 todas as novilhas foram expostas pela primeira vez à reprodução aos 24 meses de idade, ou seja, as fêmeas precoces não tiveram oportunidade de expressar seu potencial genético para a característica estudada. A essa idade, a maioria delas, provavelmente, já ultrapassou a fase da puberdade e, por esse motivo, a identificação das diferenças genéticas existentes entre os animais pode ter sido comprometida. Dessa forma, a variância genética aditiva foi baixa, a estimativa de herdabilidade foi zero e, conseqüentemente, não foi possível selecionar as mais precoces do rebanho, por não terem sido identificadas como tal.

Quando foram incluídas no arquivo as fêmeas expostas mais cedo à reprodução (IPP2), observou-se pequeno aumento na estimativa de herdabilidade da IPP, atribuído, possivelmente, ao aumento da variabilidade genética. No arquivo para IPP3, como foram incluídas apenas as fêmeas que tiveram oportunidade de concepção nas duas estações de monta, houve aumento da variabilidade genética e, conseqüentemente, da estimativa de herdabilidade para a característica, o que permitiu a identificação dos animais mais precoces. As estimativas de herdabilidade foram superiores às obtidas nos arquivos IPP1 e IPP2, apesar de ainda serem baixas.

Quando se trabalha com IPP, apenas as fêmeas que pariram fazem parte das análises, dessa forma, parte da variação existente para a característica não pode ser estimada. Outro fator que pode ter contribuído para a baixa estimativa de herdabilidade da IPP foi a curta duração da estação de monta, em torno de 60 dias. Se fosse permitido às fêmeas entrarem em reprodução a partir dos 14 meses de idade e com estação de monta mais longa, talvez fossem evidenciadas maiores diferenças genéticas entre os animais. Para IPP2 e IPP3 as estimativas de herdabilidade para IPP foram próximas às obtidas por Martins Filho e Lôbo (1991) e Garnero et al. (1999) na raça Nelore, 0,19 e 0,15 , respectivamente.

A inclusão de todas as fêmeas nascidas no rebanho (IPP4) resultou em aumento da variabilidade fenotípica e genética e, como conseqüência, aumento da estimativa de herdabilidade da IPP. O aumento da variância genética, nesse caso, pode ser explicado pelo fato de não ter ocorrido perdas de variabilidade genética atribuída à seleção. O único critério de descarte de fêmeas adotado na fazenda penaliza aquelas com problemas reprodutivos, assim, praticamente todas as fêmeas nascidas no rebanho fizeram parte desse arquivo de dados. A 
herdabilidade estimada para esse grupo foi superior à dos demais grupos, indicando que a idade ao primeiro parto é uma característica que, provavelmente, responde à seleção.

Os resultados obtidos permitem reflexões na maneira de medir e de analisar a IPP. O manejo reprodutivo tem influência nas estimativas dos componentes de variância e, conseqüentemente, nas avaliações genéticas para características reprodutivas. A adoção de estações de monta alternativas para expor as fêmeas mais cedo à reprodução pode aumentar o custo de produção da novilha, contudo, ele será compensado com os ganhos na antecipação do início da vida reprodutiva.

\section{CONCLUSÕES}

$\mathrm{Na}$ seleção para idade ao primeiro parto é importante que as novilhas sejam expostas mais cedo à reprodução. Desafiar as novilhas em idades mais jovens requer manejo adequado da fazenda, entretanto, essa prática poderá trazer benefícios com relação à seleção para precocidade sexual do rebanho, melhorando sua eficiência produtiva. A inclusão de todas as fêmeas nascidas no rebanho proporciona aumento da variabilidade genética para a idade ao primeiro parto, incrementando a estimativa de herdabilidade para a característica.

\section{REFERÊNCIAS BIBLIOGRÁFICAS}

BOLDMAN, K.G.; KRIESE, L.A.; VAN VLECK, L.D. A manual for use of MTDFREML. Clay Center: USDA-ARS, 1993. 120p.

BOURDON, R.M.; BRINKS, J.S. Genetic environmental and phenotypic relationships among gestation length, birth weight growth traits and age at first calving in beef cattle. J. Anim. Sci. v.55, p. 543-549, 1982.

BRINKS, J.S.; FIELDS, M.J.; SANDS, R.S. Relationship of scrotal circumference to puberty and subsequent reprodutive performance in male and female offspring. In: FIELDS, M.J.; SANDS, R.S. Factors affecting calf crop. Boca Raton: CRC, 1984. p.363-370.
ELER, J.P.; SILVA, J.A. II V.; FERRAZ, J.B.S. et al. Genetic evaluation of the probability of pregnancy at 14 months for Nelore heifers. J. Anim. Sci., v.80, p.951-954, 2002.

GARNERO, A.V. Estimativas de parâmetros genéticos de características reprodutivas na raça Nelore. In: REUNIÃO ANUAL DA SOCIEDADE BRASILEIRA DE ZOOTECNIA, 36., 1999, Porto Alegre. Anais... Porto Alegre: SBZ, 1999.

GRESSLER, S.L. Estudo de fatores de ambiente e parâmetros genéticos de algumas características reprodutivas em animais da raça Nelore. 1998. 149f. Dissertação (Mestrado em Zootecnia) Escola de Veterinária, Universidade Federal de Minas Gerais, Belo Horizonte.

MARTINS FILHO, R.; LÔBO, R.B Estimates of genetic correlations between sire scrotal circumference and offspring age at first calving in Nelore cattle. Rev. Bras. Genet., v.14, p.209-212, 1991

MERCADANTE, M.E.Z. Estudo das relações genético-quantitativas entre características de reprodução, crescimento e produção em fêmeas da raça Nelore. 1995. 96f. Dissertação (Mestrado) Faculdade de Medicina de Ribeirão Preto, Ribeirão Preto, SP.

PEREIRA, E.; ELER, J.P.; COSTA, F.A.A. et al. Análise genética da idade ao primeiro parto e do perímetro escrotal em bovinos da raça Nelore. Arq. Bras. Med. Vet Zootec., v.53, p.116-121, 2001.

PEREIRA, E.; ELER, J.P.; FERRAZ, J.B.S. Genetic analysis of reproductive traits in Nelore cattle. Pesq. Agropec. Bras., v.37, p.703-708, 2002.

SHORT, R.Y.; STAIMILLER, R.B.; BELLOWS, R.L. et al. Breeding heifers at one year of age: Biological and economic considerations. In: FIELDS, M.J.; SANDS, R.S. Factors affecting calf crop. Boca Raton: CRC, 1994. p.55-68

TEIXEIRA, R.A. Comparações bio-econômicas entre dois sistemas de produção com diferentes niveis de fertilidade em rebanho Nelore a pasto. 1997. 42f. Trabalho de Graduação. Faculdade de Ciências Agrárias e Veterinárias, Universidade Estadual Paulista, Jaboticabal, SP.

TOELLE, V. D.; ROBISON, O. W. Estimates of genetic correlations between testicular measurements and female reproductive traits in Cattle. J. Anim. Sci., v.60, p.89-100, 1985. 